\title{
Differential Effects of an Evolutionary-Based EMDR Therapy on Depression and Anxiety Symptoms: A Case Series Study
}

\author{
Valery Krupnik \\ Naval Hospital Camp Pendleton, Oceanside, California
}

\begin{abstract}
A novel evolutionary-based therapy, treating depression downhill (TDD), was designed as a specific therapy for depression as a syndrome (Krupnik, 2014) and later integrated with eye movement desensitization and reprocessing (EMDR) therapy into a combined TDD-EMDR treatment. The combined therapy integrates modified EMDR procedures into the theoretical context of TDD. These procedures are applied during the second (acceptance) stage of TDD-EMDR's three-stage protocol with the focus on acceptance of defeat/failure/loss rather than on distressing memories. Here, we report a case series of 21 military personnel diagnosed with depressive disorders, who received a course of TDD-EMDR. Eighty percent of completers $(n=15)$ did not meet the criteria of depressive disorder by the treatment's end. After 12 sessions, they showed a significant reduction on the Beck's Depression Inventory-II (BDI-II) with a large effect size $(d=2.8)$ and an increase in accepting disposition $(d=1.8)$ on the Acceptance and Action Questionnaire. Noncompleters showed similar to completers decrease of BDI-II scores at mid-treatment. We observed no statistically significant decrease of the anxiety symptoms on the Beck's Anxiety Inventory. These results suggest that TDD-EMDR may be an effective treatment for depressive disorders. They also indicate that it may preferentially target depressive over anxiety symptoms, as was previously observed for TDD. Suggestions are made for future research.
\end{abstract}

Keywords: depression; treating depression downhill-eye movement desensitization and reprocessing (TDD-EMDRx); treatment specificity; acceptance; evolution; military

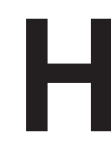
istorically, psychosocial interventions were not designed for specific disorders but rather targeted noxious patterns of thought and behavior. Psychoanalysis was originally designed to treat neuroses (Freud, 1966), whereas behavioral therapies targeted maladaptive information processing and resulting behaviors (Franks \& Wilson, 1974). Mental disorders, however, are commonly classified as symptom constellations (Diagnostic and Statistical Manual of Mental Disorder, 5th ed.; DSM-5; American Psychiatric Association, 2013); hence, by design, psychotherapies were transdiagnostic. The general trend among contemporary therapies has been toward universality rather than specificity. The most studied therapy, cognitive-behavioral therapy (CBT) was initially developed for depressive disorders but has since been used for a wide variety of conditions. Its low specificity (and high universality) has been highlighted in several reviews (Butler, Chapman, Forman, \& Beck, 2006; Haby, Donnelly, Corry, \& Vos, 2006; Hollon, Thase, \& Markowitz, 2002; Parker \& Fletcher, 2007; Shedler, 2010). These reviews reflect on the low specificity of other therapies as well. Likewise, eye movement desensitization and reprocessing (EMDR) therapy was initially developed for trauma-related disorders (Shapiro, 1989, 2001) but has been extended to a variety of afflictions including affective, personality, addiction, eating, and psychosomatic disorders (Marcus, 2008; Miller, 2010; Mosquera, Leeds, \& Gonzalez, 2014; Shapiro, 2009). Transdiagnostic therapies have also been a late trend meant to cut across DSM classification (for review, see Craske, 2012).

Universality of a tool may become a liability against its effectiveness. Lack of disorder-treatment 
specificity has been suggested as one of the factors limiting the efficacy of treating depression, and a new "choose horses for courses" paradigm has been advocated (Parker \& Fletcher, 2007; Parker, Roy, \& Eyers, 2003; Parker, Malhi, Crawford, \& Thase, 2005). The authors suggest choosing an approach specific to the nature of a depressive disorder, differentiating between psychotic, melancholic, secondary depression, and dysthymia.

More recently, specificity has also become part of psychotherapy evolution. For example, CBT has splintered into a family of specialized therapies including CBT-I for insomnia (Wilson et al., 2010), trauma-focused CBT (Cohen, Deblinger, Mannarino, \& Steer, 2004), cognitive processing therapy for posttraumatic stress disorder (Resick \& Schnicke, 1993). A new selfsystem therapy for depression tailors its interventions to the patient's profile of symptoms, depending on the prevalence of dysphoria versus anxiety (Strauman et al., 2013). Still, the science of specificity in psychotherapy is considered to be in its infancy (Beutler, 2011; Norcross \& Wampold, 2011), and a common methodology for designing (more) specific therapies is yet to be established.

\section{Evolutionary-Based Therapies for Depression}

One way of designing a disorder-specific therapy is to root it in a theory of that disorder, a position argued by Strauman (Strauman et al., 2013) among others. Such an approach has been taken in developing evolutionary-based therapies for depression. Evolutionary theory considers depression a systemic adaptive response to insurmountable adversity (Andrews \& Thomson, 2009; Keller \& Nesse, 2006; Nettle, 2004; Watt \& Panksepp, 2009) and identifies defeat (Gilbert \& Allan, 1998; Koolhaas et al., 1990; Price, Sloman, Gardner, Gilbert, \& Rohde, 1994) and learned helplessness (Seligman, 1972) as the core psychological constructs in depressive response. Evolutionary theory of depression is an umbrella term, including multiple theories emphasizing different aspects of depressive response with hypothesized adaptive value. The most relevant to this report is the notion that depressive response results in physiological, emotional, and behavioral withdrawal from protest against overpowering circumstances, thereby sparing the organism unnecessary and dangerous confrontation and increasing its survival chance (Gilbert \& Allan, 1998; Koolhaas et al., 1990; Seligman, 1972; Watt \& Panksepp, 2009). Accordingly, evolutionary-based therapies for depression target that adaptive response. Evolutionary-driven cognitive therapy (EDCT) maintains that mismatch between modern human environment and innate depressive reactions that developed early in hominid evolution contributes to depressive pathology (Giosan et al., 2014). It suggests correction of that mismatch as a therapeutic objective.

A novel integrative treatment, TDD-EMDR, has recently been suggested for treatment of depressive disorders (Krupnik, 2015a, Krupnik, 2015b). Although there are no enough data yet from comparative randomized controlled trials (RCTs) to make a definitive conclusion about the effectiveness of EMDR therapy for depression (Wood \& Ricketts, 2013), it has been shown effective for depression in case (Bae, Kim, \& Park, 2008; Broad \& Wheeler, 2006; Grey, 2011; Rosas Uribe et al., 2010) and controlled studies (Hase et al., 2015; Hofmann et al., 2014). In these studies, EMDR therapy was applied according to the standard, trauma-based protocol (Shapiro, 2001). In the controlled studies, therapeutic gains were achieved on average within five to eight sessions. We hypothesized that integrating EMDR interventions into TDD therapeutic frame might combine the effectiveness and speed of EMDR with the putative specificity of TDD, thereby conferring specificity for depression on the combined treatment as well. Another rationale for such integration was to make TDD more versatile because, in our practice, not all clients are either willing or capable to practice mindful meditation, the main component of acceptance stage of TDD. EMDR interventions appeared a more client-friendly substitute for mindfulness practice likely to require less effort and trigger less resistance.

On the contrary, another evolutionary-based therapy, treating depression downhill (TDD), considers depressive response adaptive for modern humans as well and prescribes an expedited completion of such response as its objective in the initial phase of therapy. Specifically, it follows Watt and Panksepp's (2009) idea that progression from protest to withdrawal is at the core of depressive response. To date, there have been no reports about treatment-disorder specificity of EDCT, but TDD does appear to preferentially target depressive disorders (Krupnik, 2014). TDD is a multi-modular treatment comprising exploratory, acceptance, and behavioral activation modules administered sequentially. The acceptance module is modeled after protest-withdrawal transition in animal models, and although there have been no component analyses yet, acceptance is posited to be TDD's essential agent of change that is depression specific (Krupnik, 2014). 


\section{Integrating TDD With EMDR}

Integration of EMDR therapy into TDD therapeutic frame resulted in changes introduced to both therapies, as described in detail elsewhere (Krupnik, 2015a). In short, the core EMDR interventions (Phases 3-8, Shapiro, 2001) were adapted to substitute mindful meditation in acceptance module of TDD, whose objective is to foster acceptance of defeat/ failure/loss. Accordingly, EMDR interventions in TDD-EMDR do not target traumatic experiences (if trauma is uncovered in the course of therapy and found central to the clinical presentation, we revert to the standard EMDR protocol). Instead, EMDR interventions target the client's protest against their defeat/failure/loss, helping transition into acceptance of them. This is accomplished by prompting the client to focus on the reality and finality of the incurred defeat/failure/loss with an emphasis on the emotional experience of it, as well as the emotional experience of accepting that finality. Consequently, there is no assessment of negative cognitions and their juxtaposition to positive ones and no attempt at transitioning from the former to latter. Nor do we use future template installation because it would be antithetical to acceptance. These changes to EMDR protocol follow from the difference between adaptive information processing (AIP), the theory behind EMDR therapy (Shapiro, 2001; Solomon \& Shapiro, 2008) and evolutionary theory of depression that is the basis of TDD. The mechanism of therapeutic change according to AIP is transformation of traumatic memories through a guided reprocessing. Based on evolutionary theory of depression, TDD does not consider all psychopathology a result of trauma and views disengagement from protest through an accepting mindset as an essential mechanism of therapeutic change in therapy of depression. Another, more obvious, change in EMDR protocol is substitution of its trauma history phase with exploratory phase of TDD. TDD is not trauma based, and its initial phase is, instead, focused on psychodynamic exploration into defeat/failure/loss.

In case studies, TDD-EMDR was effective in treating major depressive disorder (Krupnik, 2015b) and postpartum depression (Krupnik, 2015a). Herein, we report on the effects of TDD-EMDR on symptoms of anxiety and depression in patients with depressive disorders. In accordance with the theoretical basis of TDD, we hypothesized that depressive symptoms would be the primary target of TDD-EMDR, and that positive change in acceptance levels would be one of the outcomes.
TABLE 1. The Participants' Demographics

\begin{tabular}{|c|c|c|}
\hline \multirow[b]{2}{*}{ Variable } & \multicolumn{2}{|c|}{ Mean $(S D)$ or Number } \\
\hline & \multicolumn{2}{|c|}{ Completers Noncompleters } \\
\hline Age & $25(3.9)$ & $24(2.4)$ \\
\hline \multicolumn{3}{|l|}{ Sex } \\
\hline Males & 10 & 6 \\
\hline Females & 5 & 0 \\
\hline \multicolumn{3}{|l|}{ Race or ethnicity } \\
\hline White & 9 & 3 \\
\hline Hispanic & 5 & 2 \\
\hline Black & 1 & 1 \\
\hline Married & 7 & 2 \\
\hline \multicolumn{3}{|l|}{ Diagnosis } \\
\hline Major depression & 10 & 4 \\
\hline Persistent depressive disorder & 3 & 0 \\
\hline $\begin{array}{l}\text { Other specified depressive } \\
\text { disorder }\end{array}$ & 2 & 2 \\
\hline \multicolumn{3}{|l|}{ Medications } \\
\hline SSRI & 7 & 2 \\
\hline Wellbutrin & 1 & 0 \\
\hline Effexor & 1 & 0 \\
\hline Trazodone & 0 & 1 \\
\hline Remeron & 0 & 1 \\
\hline
\end{tabular}

Note. SSRI = selective serotonin reuptake inhibitor.

\section{Methods}

\section{Participants}

Participants were selected from a pool of patients treated by the study's author in a military mental health clinic. They were selected over the span of 3 years. The inclusion criteria were a primary diagnosis of a depressive disorder and consent to TDD-EMDR therapy. The exclusion criteria were co-occurring anxiety disorders, trauma- and stressor-related disorders (as defined in DSM-5), personality disorders, and ongoing substance abuse. Patients who were engaged in legal or administrative proceedings were also excluded to avoid interference from secondary gains. All participants were diagnosed by their treating therapist (same as the study's author). Those who received medications had their diagnosis confirmed by the treating psychiatrist. Of 21 selected patients, 15 completed the course of therapy, and the rest dropped out after the mid-treatment assessment. Their demographics are presented in Table 1. The highest level of education for all of them was high school. The breakdown of the diagnoses and medications is shown in Table 1. The patients received 12 sessions of TDD-EMDR therapy (noncompleters 
dropped out at mid-treatment, after 4-10 sessions) from the same therapist, who administered self-report measures at pretreatment (1st session; not counting the intake interview), mid-treatment (6th session), and post-treatment (12th session).

\section{Therapy}

Therapy was conducted as previously described (Krupnik, 2015a). In brief, it followed the modular structure of TDD, proceeding through its three stages: exploratory, acceptance, and behavioral activation (Krupnik, 2014). In exploratory phase, usually three to four sessions, the participants explored their experience of failure/defeat/loss believed to be the main contributors to their depression, as well as developmental experiences that could have contributed to their vulnerability. Such exploration was followed by acceptance phase. In TDD, during this phase, acceptance of defeat/failure/loss is fostered through mindful attention to their experience and meaning. TDD-EMDR differs from TDD by using EMDR interventions instead of mindful meditation. The EMDR component of TDD-EMDR differs from the standard EMDR in several ways. Unlike the standard protocol, the main target is not as much memories of particular experiences and events (they usually come up and are discussed in the exploration phase) as the emotional and cognitive experience of defeat/failure/loss as they are felt in the moment (Krupnik, 2015a). The patients are prompted to face the reality and irreversibility of what happened to them as well as their sense of helplessness. Then they are asked whether they can accept the experience and, if not, what they can do about it. The subjective degree of their acceptance is then assessed on a 1-7 Likert scale, and the experience of acceptance is processed. Obstacles to acceptance and the experience of resisting it, if any, are then processed, and the clients are directed back to face the irreversibility of their defeat/failure/loss. Such resistance is understood as an indication of the protesting mindset (which is antithetical to acceptance), and its level is assessed by the subjective intensity of feelings associated with it on a $0-10$ Likert scale. These feelings often include anger, resentment, shame, and guilt. The processing continues until acceptance reaches its ecological validity and is aided by bilateral stimulation (BLS). The length of this phase was usually four to five sessions. Then, behavioral activation was added from sessions 6 to 8 and continued through the end of therapy. In this phase, participants identified easily accessible pleasurable or comforting activities, and their engagement in them was monitored and addressed in session. Failure, if any, to engage in scheduled activities was analyzed and, if needed, more exploration and acceptance interventions were applied.

\section{Measures}

The administered measures included the Beck's Depression Inventory (BDI-II) and the Beck's Anxiety Inventory (BAI). Acceptance and Action Questionnaire (AAQ) was used to assess the levels of accepting disposition.

BDI-II is a multiple-choice questionnaire consisting of 21 items representing different symptoms of depression rated by severity, where scores of $0-13$, $14-19,20-28$, and 29-63 are considered minimal, mild, moderate, and severe, respectively. It was shown to have a high test-retest reliability of $r=.93$ and internal consistency of $\alpha=.91$ (Beck, Steer, $\&$ Brown, 1996). BAI is also a 21 -item questionnaire rated by severity of anxiety symptoms, where scores of $0-7$, $815,16-25$, and 26-63 are considered minimal, mild, moderate, and severe, respectively. Its test-retest reliability is $r=.75$ and internal consistency is $\alpha=.92$ (Beck \& Steer, 1990). Both scales are scored by adding the scores for individual items, thus yielding an overall symptom severity value.

We used the 49-item version of AAQ, a self-report questionnaire designed to measure avoidant disposition. Its test-retest reliability was estimated at $r=.65$ and internal consistency at $\alpha=.70$ (Hayes et al., 2004).

\section{Results}

\section{Treatment Outcomes}

Table 2 shows the dynamics of the participants' scores on measures of anxiety, depression, and accepting attitude. Change in pre-, mid-, and post-treatment scores was analyzed with repeated-measures analysis of variance. There was significant decrease over the course of treatment in BDI-II scores $(F[2,12]=26.30$, $p<.0005)$, and no significant difference in BAI scores $(F[2,12]=2.92, p=.08)$. The decrease of BDI-II scores from pretreatment was significant both at mid- and post-treatment, $p<.05$, but not significant between mid- and post-treatment, $p>.05$. There was also significant increase of AAQ scores at post-treatment, $F(2,12)=11.6, p<.05$, but not between mid- and post-treatment, $p>.05$. Pre-post comparison yielded large effect sizes for BDI-II and AAQ (Table 2). No significant difference was found between the medicated and not medicated sub-samples on any of the measures $(p>.6)$. Overall, TDD-EMDR was an 


\begin{tabular}{|c|c|c|c|c|c|c|c|c|}
\hline \multirow[b]{3}{*}{$\begin{array}{l}\text { Self-Report } \\
\text { Measures }\end{array}$} & \multicolumn{5}{|c|}{ Completers $(n=15)$} & \multicolumn{3}{|c|}{ Noncompleters $(n=6)$} \\
\hline & \multirow[b]{2}{*}{$\begin{array}{c}\text { Pretreatment } \\
(S D)\end{array}$} & \multirow{2}{*}{$\begin{array}{c}\text { Mid- } \\
\text { Treatment } \\
(S D)\end{array}$} & \multirow{2}{*}{$\begin{array}{c}\text { Post- } \\
\text { Treatment } \\
(S D)\end{array}$} & \multicolumn{2}{|c|}{ Effect Size $(d)^{\mathrm{a}}$} & \multirow[b]{2}{*}{$\begin{array}{c}\text { Pretreatment } \\
\qquad(S D)\end{array}$} & \multirow{2}{*}{$\begin{array}{c}\text { Mid- } \\
\text { Treatment } \\
(S D)\end{array}$} & \multirow[b]{2}{*}{ Effect Size $(d)$} \\
\hline & & & & Mid & Post & & & \\
\hline BDI-II & $32.7(7.5)$ & $16.9(8.3)^{\star}$ & $12.0(8.0)^{\star}$ & 2.1 & 2.8 & $32.8(10.1)$ & $17.2(8.7)^{\star}$ & 1.5 \\
\hline BAI & $17.7(9.2)$ & $13.2(7.5)$ & $10.6(10.4)$ & 0.5 & 0.8 & $18.3(11.4)$ & $17.8(9.0)$ & 0.04 \\
\hline AAQ & $169.5(24.6)$ & $193.1(31.3)$ & $214.0(38.7)^{\star}$ & 1.0 & 1.8 & $183.5(25.4)$ & $200.0(27.0)$ & 0.6 \\
\hline
\end{tabular}

Note. BDI-II = Beck’s Depression Inventory; BAI = Beck’s Anxiety Inventory; AAQ = Acceptance and Action Questionnaire. ${ }^{\mathrm{a}}$ Cohen's $d$ was calculated relative to the pretreatment value. ${ }^{\star} p<.05$ in comparison to pretreatment.

effective therapy in the reported sample with $80 \%$ of participants not meeting diagnostic criteria for a psychiatric disorder after 12 sessions.

In noncompleters, there was no significant difference from completers in pre- and mid-treatment scores (Table 2). Depression symptoms, as reflected on BDI-II, decreased significantly by mid-treatment with a large effect size, whereas no statistically significant change was observed in accepting attitude and anxiety symptoms (Table 2).

None of the participants manifested any adverse effects during the treatment. The mean BDI-II scores were in the severe range at pretreatment (Table 2). At the end of therapy, eight participants had BDI-II scores within the minimal range (1-14), nine were within the mild range (15-20), and four remained within the moderate range (20-29).

\section{Follow-Up}

Follow-up information was available for 9 of the 15 completers and for two of the six noncompleters. The rest had retired from the military. Six months in-person follow-up was conducted with five completers. One had a BDI-II score of 28 and remained in partial remission, able to meet her full responsibilities at work and as a single mother. Despite her continuing symptoms, she chose not to return to therapy but to manage her symptoms with an antidepressant. The other four did not meet criteria for a psychiatric diagnosis. Three of them, however, were still in maintenance antidepressant therapy.

Thanks to the centralized record keeping, medical records of four additional completers were available for 2-3 years post-treatment. Three of them did not have any mental health treatment episodes on record, and the fourth had a successful 2-month long medication and supportive therapy for postpartum depression in a context of marital stress. Thus, out of nine participants available for long-term follow-up observation (either in person or through medical records), only one chose to return to therapy although four continued taking antidepressants.

Among the six noncompleters, medical records of two participants were available for 1 and 2 years post-treatment. They did not show any mental health encounters. The other noncompleters had to interrupt their care due to retirement, and no follow-up information about them was available. In aggregate, the follow-up observation indicates a possibility of long-term gains after TDD-EMDR because of only 1 out of 11 participants available for follow-up was documented to relapse into depression and to return to therapy.

\section{Case Examples}

Below are excerpts from the transcripts of EMDR or acceptance sessions with several participants. The excerpts are given with brief case descriptions to illustrate the typical acceptance-focused intervention of TDD-EMDR therapy. Detailed case studies can be found elsewhere (Krupnik, 2015a; 2015b). In all examples, BLS was a set of about 25-30 saccadic eye movements guided by the therapist's fingertips. "C" stands for client, and " $T$ " for therapist.

We find it important to underscore that the verbal exchanges below only illustrate the cognitive shift toward acceptance, leaving the clients' emotional reaction out of frame. In our practice, the cognitive shift is often accompanied by an abreaction-like mostly nonverbal affective experience characterized by mix of sadness and relief. This experience then becomes the focus of attention in further processing until the subjective sense of acceptance reaches ecological validity (analogous to the validity of positive cognition in the standard EMDR protocol).

Client 1. The client is a 27-year-old divorced man of Native American heritage. He served one deployment 
to a war zone 5 years before the current treatment episode. He presented with complaints of insomnia, fluctuating appetite, daily depressed mood, poor concentration, loss of interest, motivation, and pleasure, feeling worthless, fatigue, and low libido. He met the criteria and was diagnosed with major depressive disorder, mild, single episode (DSM-5) by his therapist and psychiatrist. Previously, he was diagnosed with ADHD. During the exploration phase, it was revealed that one of the main chronic stressors contributing to the client's depression was his failing romantic relationship. It fostered a sense of defeat and inadequacy in him that triggered his memories of feeling unimportant and abandoned, when his mother lost custody of him due to drug use and inability to care for her children. The client's father abandoned the family early on and never had a relationship with him. The excerpt below is from a session, where the client was processing his feeling of failure as a person because of his perceived inability to win other people's (his coworkers in particular) respect and positive regard.

C: I feel ashamed. I don't even have to do that much.

$\mathrm{T}$ : How intense is the feeling on a scale from 0 to 10 ?

C: Eight.

T: Go with it.

BLS

$\mathrm{T}$ : What are you thinking now?

C: I don't like myself. I'm not right.

$\mathrm{T}$ : Is there anything you can do about it?

C: I can't do anything about it.

T: Go with it.

BLS

$\mathrm{T}$ : What are you getting?

C: I don't need people to like me. I just need to

be able to do what I need to do.

After this session, the client appeared less preoccupied with his perceived failures and more accepting of his shortcomings. His self-appraisal sounded less absolute and more related to the context of his life situation and goals.

Later in therapy, the client revealed another reason for deeply held shame. During his combat deployment, he had a "breakdown" and was left behind the wire, while his platoon went on convoy. In the excerpt below, he processes that feeling of shame.

C: I remember doing dishes while everybody was out there.

BLS

C: I started not caring about anything.
BLS

$\mathrm{T}$ : What are you getting now?

C: I was thinking about what I wanted to do when I come back.

BLS

C: I was thinking about making it home.

BLS

T: What are you getting now?

C: I feel ashamed.

T: What can you do about it now?

C: I talked to other guys. They all thought like that.

T: Go with it.

BLS

T: What are you thinking now?

C: I wonder if the guys I was with felt the way

I did.

T: Go with it.

BLS

T: What are you thinking now?

C: I know I wasn't the only one but I feel like I

was.

T: Go with it.

BLS

C: It's OK to feel like that.

T: Go with it.

BLS

C: I wasn't the only one thinking this way.

After that session, the client still was not proud of his bearing during the deployment, however, he was not seeing himself exclusively through the lens of his shame any longer. He considered his behavior a weakness and failure but did not feel anymore that they were defining him as a person. From that point, his cognitive style was growing more future oriented. This client was available for follow-up a year after the therapy. He was still taking an antidepressant but did not meet the criteria of a depressive disorder. He successfully completed his military contract, was in a happy relationship, and started college.

Client 2. The client grew up in abject poverty. His father died, when he was aged 1 year, and his mother was not stable due to drug use and intermittent employment. The client always dreamt of having a stable family of his own, which made him perceive his divorce as a personal defeat. In the excerpt below, the client processes his sense of failing his ex-wife. Noteworthy, his accepting attitude emerges without a direct prompt or intervention by the therapist. This indicates that when 
processing is set up appropriately, accepting disposition may emerge spontaneously.

$\mathrm{T}$ : What are you getting now?

C: I remember sleeping in the next room to my

wife.

T: Go with it.

BLS

$\mathrm{T}$ : What are you thinking now?

C: There was a lot of time to think, lying there.

BLS

T: What are you getting now?

C: Why wasn't I good enough for her?

T: Why weren't you?

BLS

T: What are you thinking now?

C: Sometimes things don't work out.

T: Go with it.

BLS

T: What are you getting now?

C: I wasn't really happy with my wife. I just got used to that life.

T: Go with it.

BLS

T: What are you getting now?

C: My daughter. She makes me happy.

T: Go with it.

BLS

T: What are you getting now?

C: I get my daughter in a week. Feel super-happy about it.

After this session, the client increased his social and recreational activities in line with the behavioral activation phase of therapy. Many of these activities were related to making arrangements and plans for his daughter's visit. By the end of the therapy, the client was asymptomatic. He was about to complete his military contract, secured a job, and was planning on going to college part-time.

Client 3. The client is a 30-year-old married Hispanic man with one stepchild. He presented with increasing depressive symptoms in a context of marital stress. On returning from deployment (no combat experience), he was upset by the wife's platonic friendship with another man. That revelation precipitated further decrease in his self-esteem that had always been low. He admitted that he was not very happy with his marriage, finding his wife aloof and not appreciating of him. The client's symptoms included persistent sad or irritable mood, excessive stress-related anxiety, insomnia, fluctuating appetite, loss of interest and motivation, poor concentration, anhedonia, fatigue, feeling worthless and guilty, recurrent passive death wishes. The exploration phase revealed a troubled childhood, growing up as a small boy always picked on by bigger boys. He remembered often feeling helpless and isolated, not knowing how to fight back and feeling ashamed of it. He stated that he had never felt "completely happy" and had gone through episodes of depression. Looking for protection and sense of belonging, the client joined a street gang for about 2 years, of which he felt ashamed to this day. His hair started thinning in his 20s, and by 30 , he had a visibly receded hairline and that too was a reason to feel ashamed along with his short stature.

The client met the criteria and was diagnosed with major depressive disorder, recurrent, mild by his therapist. He did not want to take psychotropic medications and declined and offer of evaluation for a trial of antidepressant. The excerpt below is from a session, where the client was processing his physical shortcomings and the resulting sense of marginalization.

T: What are you thinking?

C: Being a small boy. Having to defend myself.

T: Go with it.

BLS

$\mathrm{T}$ : What are you thinking now?

C: The urge to fit in.

T: Go with it.

BLS

T: What are you getting?

C: Feeling sad.

T: Go with it.

BLS

T: What are you getting?

C: Anger. I think about what I am missing other

than my size.

T: Go with it.

BLS

T: What are you thinking now?

C: I wish I would change back then.

T: Go with that.

BLS

T: What are you getting?

C: If I didn't care, things would be better for me.

BLS

C: But I wanted to rebel.

BLS

C: I needed to be fearless.

$\mathrm{T}$ : That was then. What can you do about it now?

C: I shouldn't let it bother me.

T: Go with it.

BLS 
T: What are you getting now?

C: There are good things about me.

T: Go with that.

BLS

T: What are you getting now?

C: I am ready to accept. . .

Despite the last statement, the client remained ambivalent about accepting his limitations. He continued processing his ambivalence in the following sessions, gradually coming to acceptance of natural feeling of fear, of the reality that there are people stronger than him, and that he may not win every fight or confrontation.

This client was unavailable for in-person follow-up. Three years later, his medical record showed advancement in rank and no recurring episodes of mental health treatment. He did, however, have a psychological evaluation for a special duty. This evaluation mentioned the dissolution of his marriage $s$ the wife's infidelity, but established his psychological stability and cleared him for the duty. Apparently, in his post-treatment years, the client remained stable and resilient.

\section{Discussion}

\section{Treatment Outcome}

In this study, we followed the dynamics of depressive and anxiety symptoms in patients suffering from depressive disorders, as they went through 12 sessions of TDD-EMDR therapy. At the treatment's end, 12 out of 15 clients no longer met diagnostic criteria for a depressive disorder, and the mean BDI-II score decreased $63 \%$ (Table 2). The decrease constitutes a large effect size of 2.8 , although without a control group, this result should be interpreted with caution. Noteworthy, most therapeutic gains came by mid-treatment (six sessions) because we did not observe statistically significant mid- to post-treatment differences on any of the measures. This finding is consistent with a notion of negative acceleration of dose-effect curve in psychotherapy (Baldwin, Berkeljon, Atkins, Olsen, $\&$ Nielsen, 2009) although the validity of such notion has recently been challenged (Falkenström, Josefsson, Berggren, \& Holmqvist, 2016). These results give a preliminary indication that TDD-EMDR may be effective for depressive disorders, both as a stand-alone treatment and in conjunction with antidepressants because we found no difference in outcome between participants who did and did not take medications.

Aside from earlier- mentioned single-case studies, the efficacy of the standard trauma-focused EMDR protocol for depression was tested in RCT. It was tested as a stand-alone treatment compared with a wait list control (Gauhar, 2016), as an adjunct therapy to CBT (Hofmann et al., 2014), and as an adjunct therapy to "treatment as usual," consisting of individual psychodynamic and group therapy in an inpatient milieu (Hase et al., 2015). In all these RCTs, EMDR was an effective therapy. The number of EMDR sessions in these studies varied from an average of five to eight, which is shorter than the 12 sessions of TDD-EMDR. However, in this study, the most effect of TDD-EMDR was also observed by mid-treatment (Table 2). The length of treatment is a significant factor in choice of therapy. Not only a shorter therapy can decrease the amount of suffering, but it may also reduce the attrition rate ( $29 \%$ in this study).

Recurrence is common in depression (Solomon et al., 2000; Wakefield \& Schmitz, 2013), which presents a challenge for therapeutic gains to last. An effort is underway to develop therapies for prevention of onset and recurrence of depressive episodes. Among treatments found effective for prevention are interpersonal therapy, CBT, and mindfulness-based cognitive therapy (Cuijpers, 2017). However, Cuijpers cautions that a limited follow-up time in most studies makes it difficult to distinguish relapse prevention from relapse delay. Therefore, long-term outcome of any therapy for depression is of special interest. For a recent in-depth discussion of long-term effects of CBT see French et al. (2017). In the present study, the follow-up observation, limited as it is, suggests a possibility of long-term gains after TDD-EMDR. Of four participants, whose medical records were followed over 2-3 years, only one had a recorded episode of depression relapse. The mentioned RCTs also noted maintenance of therapeutic gains at 3-month follow-up (Gauhar, 2016) and over a year (Hase et al., 2015).

In aggregate, it appears that the standard EMDR and TDD-EMDR have similar beneficial effects on people with depressive disorders. This raises a question of the significance of the difference between the two. Further research, especially their direct comparison, should address this question.

\section{Difference in Theory Between TDD-EMDR and the Standard EMDR}

Change in the standard EMDR protocol, as it is combined with TDD, comes from the different perspective on psychopathology. Our view of depressive disorder as an arrested adaptive depressive response has been emphasized before (Krupnik, 2015a; 2015b). More importantly, we regard a 
depressed individual not as traumatized but defeated. Of course, people can be both defeated and traumatized; nevertheless, we posit that the two are qualitatively different psychological phenomena, which is a departure from the view of suffering as a degree of trauma (from "small trauma" to "big trauma"). An in-depth discussion of this distinction, although pertinent and important, is beyond the scope of this report and should be carried out elsewhere. Here, we want to note that this theoretical difference determined the switch from helping transition from positive to negative cognitions, as done in the standard EMDR, to helping transition from protest to acceptance, as done in TDD-EMDR. We do not regard an accepting disposition as either positive or negative. Nor do we consider it a mere cognitive shift, but a full-fledged affective experience. It often comes with the feeling of sadness that we do not target for change, but for mindful awareness, another difference from the standard protocol, where the objective of the desensitization phase is to decrease the subjective distress to zero (Shapiro, 2001).

In accord with our theoretical position, we consider acceptance a putative mechanism of change in the second (acceptance) phase of TDD and TDD-EMDR (Krupnik, 2014). Single-case studies of TDD-EMDR showed increase of acceptance, as measured by AAQ, in the course of therapy (Krupnik, 2015a; 2015b). Here, we confirm this observation, showing a significant increase of acceptance in our sample (Table 2).

We need to underscore that in TDD, acceptance is conceptualized and practiced differently from acceptance and commitment therapy (ACT), where acceptance also is a central component. In ACT, clients are encouraged to accept their thoughts and feelings as internal events and to stop avoiding them (Hayes, Strosahl, \& Wilson, 1999), whereas in TDD and TDD-EMDR, acceptance is practiced more literally, similarly to learned helplessness (Seligman, 1972), and in a more prescriptive fashion. The goal is to facilitate the disengagement from protest through acceptance of the defeat/failure/loss.

\section{Symptom Specificity of the Outcome}

As expected, depressive symptoms appeared more responsive than anxiety to TDD-EMDR (Table 2). This is consistent with the earlier observation on TDD (Krupnik, 2014). Many interventions are close in efficacy for both anxiety and depressive symptoms, including selective serotonin reuptake inhibitors (Sugarman, Loree, Baltes, Grekin, \& Kirsch, 2014) and CBT (Butler et al., 2006). An interesting exception is mindfulness-based therapy, which has differential effects on anxiety and depressive symptoms depending on the clinical context, that is, anxiety versus depressive disorder, as revealed in a meta-analytic study (Hofmann, Sawyer, Witt, \& Oh, 2010). The authors established that although mindfulness-based therapy had large to moderate effect size on symptoms of anxiety in patients with anxiety, pain disorders, and cancer, it had no significant effect on anxiety in patients with depression. The most recent meta-analysis of ACT's efficacy suggests a possibility that ACT, too, may be more efficacious for depression than for anxiety (Hacker, Stone, \& MacBeth, 2016).

Regarding the preferential effect on depressive symptoms in this study, we cannot rule out a "ceiling" effect because at pre-treatment (Table 2), the mean scores for depression were significantly higher (in the severe range on BDI-II scale) than for anxiety (in the moderate range on BAI scale). Still, TDD/TDD-EMDR specificity for depression remains a possibility.

The question of symptom-intervention specificity may appear of little relevance. After all, therapies are meant to treat individuals and their afflictions, not the individual symptoms. It is, however, relevant to understanding the therapeutic mechanisms, to developing new therapies in accordance with that understanding, and to matching interventions with individuals or, as Parker put it, "choosing horses for courses" (Parker et al., 2005).

\section{Future Research}

An important next step in research of TDD-EMDR would be a RCT with at least three comparison treatments: the standard EMDR protocol, TDD, and intent-to-treat control; preferably, evidence-based therapies such as ACT, CBT, interpersonal, behavioral activation, and brief psychodynamic therapy should be included. The hypothesized role of acceptance as an agent of change could be tested in a component/ dismantling study as well as by mediation analysis. The latter could be carried out within an RCT. We do not exclude a possibility that the standard EMDR can also foster accepting disposition because coming to terms with trauma means a more accepting attitude to the traumatic event and the self.

A different facet of comparing TDD-EMDR to the standard protocol is a possibility that the two therapies may be differentially effective for different subpopulations of depressed people. For example, the standard EMDR may prove more effective for people with higher levels of traumatization, which can be addressed using a traumatization checklist as in Gauhar (2016). 
Another area of inquiry is possible mutual dependence of anxiety and depression symptoms. For example, regulatory focus theory (Higgins, 1998; Klenk, Strauman, \& Higgins, 2011) posits two main strategies used in adversity: anxiety and depressive responses regulated by prevention and promotion systems, respectively. Downregulation of promotion system concomitant with upregulation of prevention system is hypothesized to lead to co-occurrence of depressive and anxiety symptoms. Certain interventions may be more effective in targeting either one or the other system and should be matched accordingly. Strauman et al. have implemented such approach in their self-system therapy that is based on regulatory focus theory (Strauman et al., 2013). In a follow-up study (Strauman et al., 2015), the authors demonstrated a preferential effect of interventions, targeting promotion system on symptoms of depression versus anxiety. It would be interesting to test how each module of TDD-EMDR (and TDD), as well as the whole therapy, affect promotion and prevention systems.

\section{Limitations}

Among the limitations of this study is its case series design, which did not control for other causes of improvement such as passage of time, placebo effect, and nonspecific factors associated with therapeutic relationship, thus preventing conclusions about causation. The assumed effects of EMDR interventions on acceptance as well as the role of acceptance in the outcome remain untested. The small sample size and few participants available for long-term follow-up limit generalization of the findings. Another limitation concerns the sample's demographic homogeneity (Table 1): all patients were relatively young people of similar socioeconomic status; as military personnel, they were facing similar stressors and challenges in a highly structured environment, both stressful and supportive. It is, therefore, unclear how the observed effects may generalize to different demographics. Still, our sample represents the ethnic diversity of the US military and general population, including its major ethnicities (Table 1).

Having all patients assessed and treated by the author of the study may have contributed to the consistence of treatment delivery, but also had a potential of introducing a bias. Despite these limitations, the results lay the groundwork for further study of TDD-EMDR use in therapy of depressive disorders.

\section{References}

American Psychiatric Association. (2013). Diagnostic and statistical manual of mental disorders (5th ed.). Arlington, VA: American Psychiatric Publishing.

Andrews, P. W., \& Thomson, J. A. (2009). The bright side of being blue: Depression as an adaptation for analyzing complex problems. Psychol Review, 116(3), 620-654. http: / / dx.doi.org/10.1037 / a0016242

Bae, H., Kim, D., \& Park, Y. C. (2008). Eye movement desensitization and reprocessing for adolescent depression. Psychiatry Investigation, 5(1), 60-65. http:// dx.doi. org/10.4306/pi.2008.5.1.60

Baldwin, S. A., Berkeljon, A., Atkins, D. C., Olsen, J. A., \& Nielsen, S. L. (2009). Rates of change in naturalistic psychotherapy: Contrasting dose-effect and goodenough level models of change. Journal of Consulting and Clinical Psychology, 77(2), 203-211. http:/ / dx.doi.org/ 10. 1037 / a0015235

Beck, A. T., \& Steer, R. A. (1990). Manual for the beck anxiety inventory. San Antonio, TX: The Psychological Corporation.

Beck, A. T., Steer, R. A., \& Brown, G. K. (1996). Manual for the beck depression inventory-II. San Antonio, TX: The Psychological Corporation.

Beutler, L. E. (2011). Prescriptive matching and systematic treatment selection. In J. C. Norcross, G. R. VandenBos, \& D. K. Freedheim (Eds.), History of psychotherapy: Continuity and change (2nd ed., pp. 402-417). Washington, DC: American Psychological Association.

Broad, R. D., \& Wheeler, K. (2006). An adult with childhood medical trauma treated with psychoanalytic psychotherapy and EMDR: A case study. Perspectives in Psychiatric Care, 42(2), 95-105. http://dx.doi.org/10. $1111 /$ j.1744-6163.2006.00058.x

Butler, A. C., Chapman, J. E., Forman, E. M., \& Beck, A. T. (2006). The empirical status of cognitive-behavioral therapy: A review of meta-analyses. Clinical Psychology Review, 26(1), 17-31. http://dx.doi.org/10.1016/j.cpr. 2005.07.003

Cohen, J. A., Deblinger, E., Mannarino, A. P., \& Steer, R. A. (2004). A multisite, randomized controlled trial for children with sexual abuse-related PTSD symptoms. Journal of the American Academy of Child \& Adolescent Psychiatry, 43(4), 393-402. http://dx.doi.org/10.1097/ 00004583-200404000-00005

Craske, M. G. (2012). Transdiagnostic treatment for anxiety and depression. Depression and Anxiety, 29(9), 749-753.

Cuijpers, P. (2017). Preventing the onset of depressive disorders. In N. L. Cohen (Ed.), Public health perspectives on depressive disorders (pp. 411-430). Baltimore, MD: John Hopkins University Press.

Falkenström, F., Josefsson, A., Berggren, T., \& Holmqvist, R. (2016). How much therapy is enough? Comparing dose-effect and good-enough models in two different settings. Psychotherapy, 53(1), 130-139. http://dx.doi. org/10.1037/pst0000039 
Franks, C. M., \& Wilson, G. T. (Eds.). (1974). Annual review of behavior therapy: Theory and practice. New York, NY: Brunner/Mazel.

French, L. R., Thomas, L., Campbell, J., Kuyken, W., Lewis, G., Williams, C., . . T Turner, K. M. (2017). Individuals' long term use of cognitive behavioural skills to manage their depression: A qualitative study. Behavioural and Cognitive Psychotherapy, 45(1), 46-57. http:/ / dx.doi.org/ $10.1017 /$ S1352465816000382

Freud, S. (1966). The complete introductory lectures on psychoanalysis (J. Strachey, Trans.). New York, NY: W. W. Norton \& Company, Inc.

Gauhar, Y. W. M. (2016). The efficacy of EMDR in the treatment of depression. Journal of EMDR Practice and Research, 10(2), 59-69. http: / / dx.doi.org/10.1891/19333196.10.2.59

Gilbert, P., \& Allan, S. (1998). The role of defeat and entrapment (arrested flight) in depression: An exploration of an evolutionary view. Psychological Medicine, 28(3), 585598. http: / / dx.doi.org/10.1017/ S0033291798006710

Giosan, C., Cobeanu, O., Mogoase, C., Muresan, V., Malta, L. S., Wyka, K., \& Szentagotai, A. (2014). Evolutionary cognitive therapy versus standard cognitive therapy for depression: A protocol for a blinded, randomized, superiority clinical trial. Trials, 15, 83. http://dx.doi.org/10. $1186 / 1745-6215-15-83$

Grey, E. (2011). A pilot study of concentrated EMDR: A brief report. Journal of EMDR Practice and Research, 5(1), 14-24. http: / / dx.doi.org/10.1891/1933-3196.5.1.14

Haby, M. M., Donnelly, M., Corry, J., \& Vos, T. (2006). Cognitive behavioural therapy for depression, panic disorder and generalized anxiety disorder: A meta-regression of factors that may predict outcome. Australian and New Zealand Journal of Psychiatry, 40(1), 9-19. http:/ / dx.doi.org/10.1080/j.1440-1614.2006. 01736.x

Hacker, T., Stone, P., \& MacBeth, A. (2016). Acceptance and commitment therapy - Do we know enough? Cumulative and sequential meta-analyses of randomized controlled trials. Journal of Affective Disorders, 190, 551-565. http:// dx.doi.org/10.1016/j.jad.2015.10. 053

Hase, M., Balmaceda, U. M., Hase, A., Lehnung, M., Tumani, V., Huchzermeier, C., \& Hofmann, A. (2015). Eye movement desensitization and reprocessing (EMDR) therapy in the treatment of depression: A matched pairs study in an inpatient setting. Brain and Behavior, 5(6), e00342. http: / / dx.doi.org/10.1002/brb3.342

Hayes, S. C., Strosahl, K., \& Wilson, K. G. (1999). Acceptance and commitment therapy: An experiential approach to behavior change. New York, NY: Guilford Press.

Hayes, S. C., Strosahl, K., Wilson, K. G., Bissett, R. T., Pistorello, J., Toarmino, D., . . McCurry, S. M, Polusny, M. A., Dykstra, T. A., \& Bond, F. W. (2004). Measuring experiential avoidance: A preliminary test of a working model. The Psychological Record, 54(4), 553-578. http:// dx.doi.org/ 10.1007/BF03395492
Higgins, E. T. (1998). Promotion and prevention: Regulatory focus as a motivational principle. Advances in Experimental Social Psychology, 30, 1-46.

Hofmann, A., Hilgers, A., Lehnung, M., Liebermann, P., Ostacoli, L., Schneider, W., \& Hase, M. (2014). Eye movement desensitization and reprocessing as an adjunctive treatment of unipolar depression: A controlled study. Journal of EMDR Practice and Research, 8(3), 103-112. http: / / dx.doi.org/ 10.1891/1933-3196.8.3.103

Hofmann, S. G., Sawyer, A. T., Witt, A. A., \& Oh, D. (2010). The effect of mindfulness-based therapy on anxiety and depression: A meta-analytic review. Journal of Consulting and Clinical Psychology, 78(2), 169-183. http://dx.doi. org/10.1037/a0018555

Hollon, S. D., Thase, M. E., \& Markowitz, J. C. (2002). Treatment and prevention of depression. Psychological Science in the Public Interest, 3(2), 39-77. http:/ / dx.doi. org/10.1111/1529-1006.00008

Keller, M. C., \& Nesse, R. M. (2006). The evolutionary significance of depressive symptoms: Different adverse situations lead to different depressive symptom patterns. Journal of Personality and Social Psychology, 91(2), 316-330. http: / / dx.doi.org/10.1037 / 0022-3514.91.2.316

Klenk, M. M., Strauman, T. J., \& Higgins, E. T. (2011). Regulatoryfocus and anxiety: A self-regulatory model of GAD-depression comorbidity. Personality and Individual Differences, 50(7), 935-943. http:// dx.doi.org/10.1016/j. paid.2010.12.003

Koolhaas, J. M., Hermann, P. M., Kemperman, C., Bohus, B., Hoofdakker, R. Hvd., \& Beersma, D. G. M. (1990). Single social interaction leading to defeat in male rats induces a gradual, but long lasting behavioral change: A model of depression? Neuroscience Research Communication, 7, 35-41.

Krupnik, V. (2014). A novel therapeutic frame for treating depression in group treating depression downhill. SAGE Open, 4(1), 215824401452379-12. http://dx.doi.org/10. $1177 / 2158244014523793$

Krupnik, V. (2015a). Integrating EMDR into a novel evolutionary-based therapy for depression: A case study of postpartum depression. Journal of EMDR Practice and Research, 9(3), 137-149. http://dx.doi.org/10.1891/ 1933-3196.9.3.137

Krupnik, V. (2015b). Integrating EMDR into an evolutionary-based therapy for depression: A case study. Clinical Case Reports, 3(5), 301-307. http:/ / dx.doi.org/10.1002/ ccr3.228

Marcus, S. V. (2008). Phase 1 of integrated EMDR an abortive treatment for migraine headaches. Journal of EMDR Practice and Research, 2(1), 15-25. http:// dx.doi.org/10. 1891/1933-3196.2.1.15

Miller, R. (2010). The feeling-state theory of impulse-control disorders and the impulse-control disorder protocol. Traumatology, 16(3), 2-10. http://dx.doi.org/10.1177/ 1534765610365912

Mosquera, D., Leeds, A. M., \& Gonzalez, A. (2014). Application of EMDR therapy for borderline personality 
disorder. Journal of EMDR Practice and Research, 8(2), 7489. http: / / dx.doi.org/10.1891/1933-3196.8.2.74

Nettle, D. (2004). Evolutionary origins of depression: A review and reformulation. Journal of Affective Disorders, 81(2), 91-102. http:// dx.doi.org/10.1016/j.jad.2003.08. 009

Norcross, J. C., \& Wampold, B. E. (2011). What works for whom: Tailoring psychotherapy to the person. Journal of Clinical Psychology, 67(2), 127-132. http:/ / dx.doi.org/ $10.1002 /$ jclp. 20764

Parker, G., \& Fletcher, K. (2007). Treating depression with the evidence-based psychotherapies: A critique of the evidence. Acta Psychiatrica Scandinavica, 115(5), 352-359. http: / / dx.doi.org/10.1111/j.1600-0447.2007.01007.x

Parker, G. B., Malhi, G. S., Crawford, J. G., \& Thase, M. E. (2005). Identifying "paradigm failures" contributing to treatment-resistant depression. Journal of Affective Disorders, 87(2-3), 185-191. http://dx.doi.org/10.1016/j.jad. 2005.02.015

Parker, G., Roy, K., \& Eyers, K. (2003). Cognitive behavior therapy for depression? Choose horses for courses. American Journal of Psychiatry, 160(5), 825-834. http:// dx.doi.org/10.1176/appi.ajp.160.5.825

Price, J., Sloman, L., Gardner, R., Gilbert, P., \& Rohde, P. (1994). The social competition hypothesis of depression. British Journal of Psychiatry, 164(3), 309-315. http:/ / dx. doi.org/10.1192/bjp.164.3.309

Resick, P. A., \& Schnicke, M. (1993). Cognitive processing therapy for rape victims: A treatment manual. Newbury Park, CA: Sage.

Rosas Uribe, M. E., López Ramírez, E. O., \& Jarero Mena, I, Uribe, M. E. R., Ramírez, E. O. L., \& Mena, I. J. (2010). Effect of the EMDR psychotherapeutic approach on emotional cognitive processing in patients with depression. The Spanish Journal of Psychology, 13(1), 396-405. http: / / dx.doi.org/10.1017/S1138741600003954

Seligman, M. E. (1972). Learned helplessness. Annual Review of Medicine, 23, 407-412. http://dx.doi.org/10. 1146/ annurev.me.23.020172.002203

Shapiro, F. (1989). Eye movement desensitization: A new treatment for post-traumatic stress disorder. Journal of Behavior Therapy and Experimental Psychiatry, 20(3), 211217. http: / / dx.doi.org/10.1016/0005-7916(89)90025-6

Shapiro, F. (2001). Eye movement desensitization and reprocessing: Basic principles, protocols and procedures (2nd ed). New York, NY: Guilford Press.

Shapiro, R. E. (2009). EMDR solutions II: For depression, eating disorders, performance, and more. New York, NY: WW Norton \& Company.

Shedler, J. (2010). The efficacy of psychodynamic psychotherapy. American Psychologist, 65(2), 98-109. http:/ /dx. doi.org/10.1037/a0018378

Solomon, D. A., Keller, M. B., Leon, A. C., Mueller, T. I., Lavori, P. W., Shea, M. T., ... Endicott, J. (2000). Multiple recurrences of major depressive disorder. American Journal of Psychiatry, 157(2), 229-233. http:/ / dx.doi.org/ 10.1176/appi.ajp.157.2.229

Solomon, R. M., \& Shapiro, F. (2008). EMDR and the adaptive information processing model potential mechanisms of change. Journal of EMDR Practice and Research, 2(4), 315-325. http:// dx.doi.org/10.1891/1933-3196.2. 4.315

Strauman, T. J., Goetz, E. L., Detloff, A. M., MacDuffie, K. E., Zaunmüller, L., \& Lutz, W. (2013). Self-regulation and mechanisms of action in psychotherapy: A theory-based translational perspective. Journal of Personality, 81(6), 542-553. http:/ / dx.doi.org/10.1111/jopy.12012

Strauman, T. J., Socolar, Y., Kwapil, L., Cornwell, J. F., Franks, B., Sehnert, S., \& Higgins, E. T. (2015). Microinterventions targeting regulatory focus and regulatory fit selectively reduce dysphoric and anxious mood. Behaviour Research and Therapy, 72, 18-29. http://dx.doi.org/ $10.1016 /$ j.brat.2015.06.003

Sugarman, M. A., Loree, A. M., Baltes, B. B., Grekin, E. R., \& Kirsch, I. (2014). The efficacy of paroxetine and placebo in treating anxiety and depression: A meta-analysis of change on the Hamilton Rating Scales. PLoS ONE, 9(8), e106337. http:/ / dx.doi.org/10.1371/journal. pone. 0106337

Wakefield, J. C., \& Schmitz, M. F. (2013). When does depression become a disorder? Using recurrence rates to evaluate the validity of proposed changes in major depression diagnostic thresholds. World Psychiatry, 12(1), 44-52. http: / / dx.doi.org/10.1002/wps.20015

Watt, D. F., \& Panksepp, J. (2009). Depression: An evolutionarily conserved mechanism to terminate separation distress? A review of aminergic, peptidergic, and neural network perspectives. Neuropsychoanalysis, 11(1), 7-51. http://dx.doi.org/10.1080/15294145. 2009.10773593

Wilson, S. J., Nutt, D. J., Alford, C., Argyropoulos, S. V., Baldwin, D. S., Bateson, A. N., . . Wade, A. G, Espie, C. (2010). British Association for Psychopharmacology consensus statement on evidence-based treatment of insomnia, parasomnias and circadian rhythm disorders. Journal of Psychopharmacology, 24(11), 1577-1601. http: / / dx.doi.org/10.1177/0269881110379307

Wood, E., \& Ricketts, T. (2013). Is EMDR an evidencedbased treatment for depression? A review of the literature. Journal of EMDR Practice and Research, 7(4), 225-235.

Acknowledgments. I want to acknowledge the editorial team's help in improving the manuscript.

Correspondence regarding this article should be directed to Valery Krupnik, PhD, Department of Mental Health, Naval Hospital Camp Pendleton, 200 Mercy Circle, Oceanside, CA 92055.E-mail: vkrupnik@gmail.com 